\title{
EXPERIMENTS ON CHUTE FLOWS OF GRANULAR MATERIALS
}

\author{
H. AHN, C.E. BRENNEN, and R.H. SABERSKY \\ California Institute of Technology, Pasadena, CA 91125 (U.S.A.)
}

\section{ABSTRACT}

Experiments on continuous, steady flows of granular materials down an inclined channel or chute were made with the object of acquiring information on the rheological properties of the granular material flow and the nature of the boundary condition on the base of the channel. Specifically measurements were made of the mean material velocities and velocity profiles on all boundaries of the flow using cross-correlation of two neighboring fibre-optic displacement probes. The output from these probes was used to obtain (1) the unsteady or random component of the particle velocity in the longitudinal direction and (2) a measure of the volume fraction of the flow in contact with the base by counting the frequency of passage of the particles. Measurement was also made of the depth of the flow, the mass flow rate and the shear stress on the base. The latter employed a strain-gauged shear force plate built into the base.

The experiments are currently in progress and so further data will be presented at a later date. Nevertheless the preliminary data have yielded a number of interesting features.

\section{INTRODUCTION}

Recent research has added greatly to our knowledge of the rheology of rapid granular flows. In particular the theoretical models (for example Jenkins and Savage [1983] and Lun et al [1984]) have led to a comprehension of how the stresses and volume fraction in a granular flow are related to the velocity gradients and to the kinetic energy associated with the random motions of the particles (the so-called granular temperature). These advances have been greatly aided by the computer simulations (e.g. Campbell and Brennen (1985a and b), Walton (1984)) which have been able to provide qualitative and quantitative information in situations in which the complexity is too great to allow evaluation with the current analytical methods. Progress in experimental methods for granular materials has been much slower, hindered by the obvious difficulties involved in trying to make point measurement of velocity, volume fraction, or granular temperature in the interior of a granular material flow.

The present paper reports on an ongoing experimental program studying the continuous, steady flows of granular materials down an inclined channel or chute. One of the objectives of this program is to acquire information on the rheological properties of the granular material flow and the nature of the boundary condition at the kind of smooth, solid walls which would be common in engineering practice. Since the program is ongoing further data will be presented at a later time. Despite its incompletness the data presented 
here has some interesting features which we thought would be of interest to this workshop.

The present state of the experimental information on granular material flows consists of a number of Couette flow studies (e.g., Savage and McKeown [1983], Hanes [1985], Craig et al [1986] and several studies of flows down inclined chutes (e.g. Bailard [1978], Augenstein and Hogg [1978], Sayed and Savage [1983]). The understandable, initial objective of some of the Couette flow experiments (such as those of Savage and McKeown) was to produce a simple shear flow with a uniform velocity gradient, uniform volume fraction and, hopefully, uniform granular temperature. Toward this end the solid containing surfaces were roughened to produce a no-slip condition. Practical engineering circumstances require us to learn how to model the conditions at smooth walls at which slip occurs. This presents some difficulties because the boundary conditions on the velocity and granular temperature at smooth walls are far from clear (see, for example Campbell and Gong [1987]). The computer simulations of chute flows with speculative "smooth wall" conditions (Campbell and Brennen [1985]) indicate a boundary layer next to the wall which has a lower volume fraction and a higher granular temperature than the material further from the wall. They also indicate a "conduction" of granular heat from this layer toward the bulk.

\section{EXPERIMENTAL MEASUREMENTS}

The experiments were carried out in a 7.62 wide $120 \mathrm{~cm}$. long rectangular alurr ınum channel or "chute" installed in a continuous flow, granular material facility previously described (Patton et al [1986]). In this paper, results are described for the flow of glass beads with a diameter, $d$, of $0.126 \mathrm{~cm}$. The flow into the channel is regulated by a vertical gate, the opening between this and the channel base being referred to as the gate height, $h_{o}$. The channel is inclined at different angles, $\theta$, to the horizontal and measurements were only taken after a steady state flow had been established.

The following measurements were taken for a range of $h_{o}$ and $\theta$. Point probes were used to record the depth, $h$, of the flow at several longitudinal locations in the channel and the mass flow rate was obtained by timed collection of material discharging from the chute. To measure the shear stress between the flowing material and the chute base, a rectangular hole, $11.4 \mathrm{~cm}$ long and $3.8 \mathrm{~cm}$ wide was cut into the chute base and replaced by a plate supported by strain-gauged flexures sensitive to the shearing force applied to the plate. Calibration of this balance was achieved by placing weights on the plate with the channel set at various inclinations. The clearance between the plate and the rest of the chute base was adjusted to be about $0.02 \mathrm{~cm}$, much smaller than the particle size. Nevertheless, dirt particles would occasionally get trapped in the gap and this necessitated cleaning of the gap prior to each measurement.

In addition to the above, a fibre-optic probe similar to that originally devised by Savage [1979] was developed to measure particle velocities on the free surface, the sidewalls and the channel base. Several of these were fabricated. They each consisted of two MTI fibre- 
optic displacement probes set with their faces flush in an aluminum plug which was, in turn, either set flush in the channel base or sidewalls or held close to the free surface of the flowing granular material. The probe faces were $0.16 \mathrm{~cm}$ in diameter and of the type in which one semi-circle of the face consisted of transmitting fibres and the other of receiving fibres. The specific geometry is shown in figure 1 . These probes were calibrated by placing them close to a revolving drum to which particles had been glued and measuring both the probe output (see below) and the drum peripheral velocity.



Figure 1. Geometry of the faces of the two displacement probes used for velocity measurements with the $0.126 \mathrm{~cm}$ diameter glass beads.

The output from each of these velocity measuring devices was processed in the following way. First the signals from each of the two displacement probes were simultaneously digitized and stored using a data acquisition system recording at $3 \times 10^{4}$ samples/sec. Except for very slow flows, the record time was about 0.5 secs; typically each record detected the passage of 300-600 particles. The two records were digitally cross-correlated over the entire record in order to obtain the mean particle velocity, $\bar{u}$. This information was then used to identify the peaks on the two records corresponding to the passage of a particular particle. Where no such correspondence could be firmly established or where the peak was below a certain threshold, the data was discarded for the purposes of this second part of the analysis. However, where positive identification was made, the velocity of that individual particle was obtained from the time interval between the peaks it generated on the two records. In this way, a set of instantaneous particle velocities were obtained and ensemble-averaging was used to obtain both the mean velocity, $\bar{u}$ and the root-mean-square of the fluctuating velocity, $u^{\prime}$. Though the latter represents only one component of the fluctuating kinetic energy, it should nevertheless be some measure of the granular temperature. Finally, the number of particles per unit time detected by the probe, $\dot{n}$, was divided by the velocity, $\bar{u}$, to obtain a linear concentration, $\nu^{*}$, of particles per unit length.

Though other measurements are being carried out as a part of the on-going program 
(including measurements of the heat transfer rates) this paper will be confined to presentation of some of the results obtained with the above equipment.

\section{PRELIMINARY OBSERVATIONS}

Early in the present program it was found that the data were quite sensitive to the cleanliness of the base and the sides of the channel. After running for some time, a thin layer of grime would be deposited on the chute by the particles and would apparently alter the surface boundary conditions. The obvious solution was regular cleaning and some of the data was obtained with this precaution. But a virtue was also made of this difficulty and data was taken with a dirty chute in order to determine those characteristics of the rheology which were sensitive to the boundary condition at the wall and those which are not sensitive. Thus, as well as presenting data for three gate openings, $h_{o}$, of $1.59 \mathrm{~cm}, 2.54$ $\mathrm{cm}$, and $5.08 \mathrm{~cm}$ and various chute inclination ranging from $\theta=15^{\circ}$ to $37^{\circ}$, data is also classified by whether the chute was "clean", "moderately dirty" or "dirty". Though these characterizations are clearly subjective, the differences and/or similarities in the data are, we believe, of significance.

Fibre-optic probe measurements were made at three lateral locations on the channel base and at four lateral locations on the free surface and at approximately 12 vertical positions on a sidewall. For brevity, details of these profiles will not be included here but will be available later. It is sufficient to note the following characteristics of the profiles. The profiles on the free surface at all speeds and on the base at higher speeds were always close to uniform. Only on the base at lower speeds was the nonuniformity due to the sidewalls significant. Under these conditions the lateral profiles appeared to be roughly parabolic with a side-wall velocity of not less than $80 \%$ of the velocity at the channel center.

The velocity profiles at the sidewall were examined with greater resolution since the gradients in velocity and solid fraction were expected to be greater in the direction normal to the base. These sidewall profiles were used as an indication of the vertical profile elsewhere in the flow, though we do not have evidence for the validity of these indications. The profiles of fluctuating velocity and linear solid fraction indicated surprisingly small variation in these quantities over the depth of the flow except within a distance of about one particle diameter from the base. Furthermore, the mean velocity gradient normal to the base, $d \bar{u} / d y$, was similarly constant outside this smaller-than-expected boundary layer. As a result of these observations, we shall assume in the remainder of this presentation that the solid fraction is characterized by the mean (or overall) solid fraction, $\nu_{m}$ (see below) and that the shear rate, $d \bar{u} / d y$, can be characterized by the difference between the base and free surface velocities, $\Delta u$, divided by the depth, $h$, of the flow.

The differences between all the velocity measurements were sufficiently small so that 
an average mean velocity, $\bar{u}_{M}$, for any of the flows could be fairly accurately obtained by taking an appropriate mean. From $\bar{u}_{M}$ and the mass flow rate $\dot{m}$, the local flow depth measurement, $h$, and the particle density, $\rho$, a mean solid fraction, $\nu_{M}$ could then be obtained as $\nu_{M}=\dot{m} / \rho h b \bar{u}_{M}$ where $b$ is the channel width. From previous experiments (Savage [1979], Sayed and Savage [1983], Bailard [1978]) and from computer simulations (Campbell and Brennen [1986b]) there are indications that the solid fraction, $\nu$, is smaller in a layer next to the base than in the bulk of the material which appears to ride on this boundary layer. The sidewall measurements mentioned above revealed a similar deficiency in the linear solid fraction near the base; however, the boundary layer seemed to be only about one particle diameter thick.

Another perspective on this boundary layer can be gained by comparing the linear concentration of particles per meter, $\nu^{*}$, detected by the fibre-optic probes with the mean solid fraction, $\nu_{m}$. If the former is roughly converted to a wall solid fraction, $\nu_{w}$, by assuming that the probe only detects a single particle layer so that $\nu_{w}=\pi\left(d \nu^{*}\right)^{3} / 6$ it transpires that $\nu_{w}$ is close to $\nu_{m}$ The largest deviation occurs for low volume fraction flows (as one would expect) where $\nu_{w}$ may be as little as one-half of $\nu_{m}$.

\section{STRESSES AND RHEOLOGICAL BEHAVIOR}

Neglecting the effects of the side wall in these relatively shallow and wide flows the normal stress, $\tau_{N}$, on the channel base is calculated as $\rho \nu_{M} g h$; as previously discussed the shear stress, $\tau_{S}$, is measured directly. The analytical work of Jenkins and Savage [1983] and Lun et al [1984] suggests that the rheological behavior of rapidly-flowing granular materials in a shearing flow such as occurs in the chute is of the form

$$
\begin{gathered}
\tau_{S} \propto g_{S}(\nu) \rho d\left(\frac{d \bar{u}}{d y}\right) c^{\prime} \\
\tau_{N} \propto g_{N}(\nu) \rho\left(c^{\prime}\right)^{2}
\end{gathered}
$$

where $g_{S}(\nu), g_{N}(\nu)$ are functions of the solid fraction (which differ somewhat depending on the analytical approach), $c^{r}$ is the root mean square of the velocity fluctuations and $d \bar{u} / d y$ is the mean velocity gradient. Here, an attempt will be made to compare these relations with the experimental measurements. Though there are indications that the random fluctuations may be nonisotropic, particularly. at denser packings (Campbell and Brennen [1985a]) we shall assume isotropy and therefore that $\left(c^{\prime}\right)^{2}=3\left(u^{\prime}\right)^{2}$.

First, in figure 2, we plot the experimental values of

$$
g_{S}(\nu)=\tau_{S} / \rho d \frac{\Delta u}{h}(u)^{\prime}
$$

versus the mean solid fraction, $\nu_{m}$. As can be observed, all of the data obtained to date appears to conform to a single function $g_{s}(\nu)$ over a wide range of velocities (inclinations) 


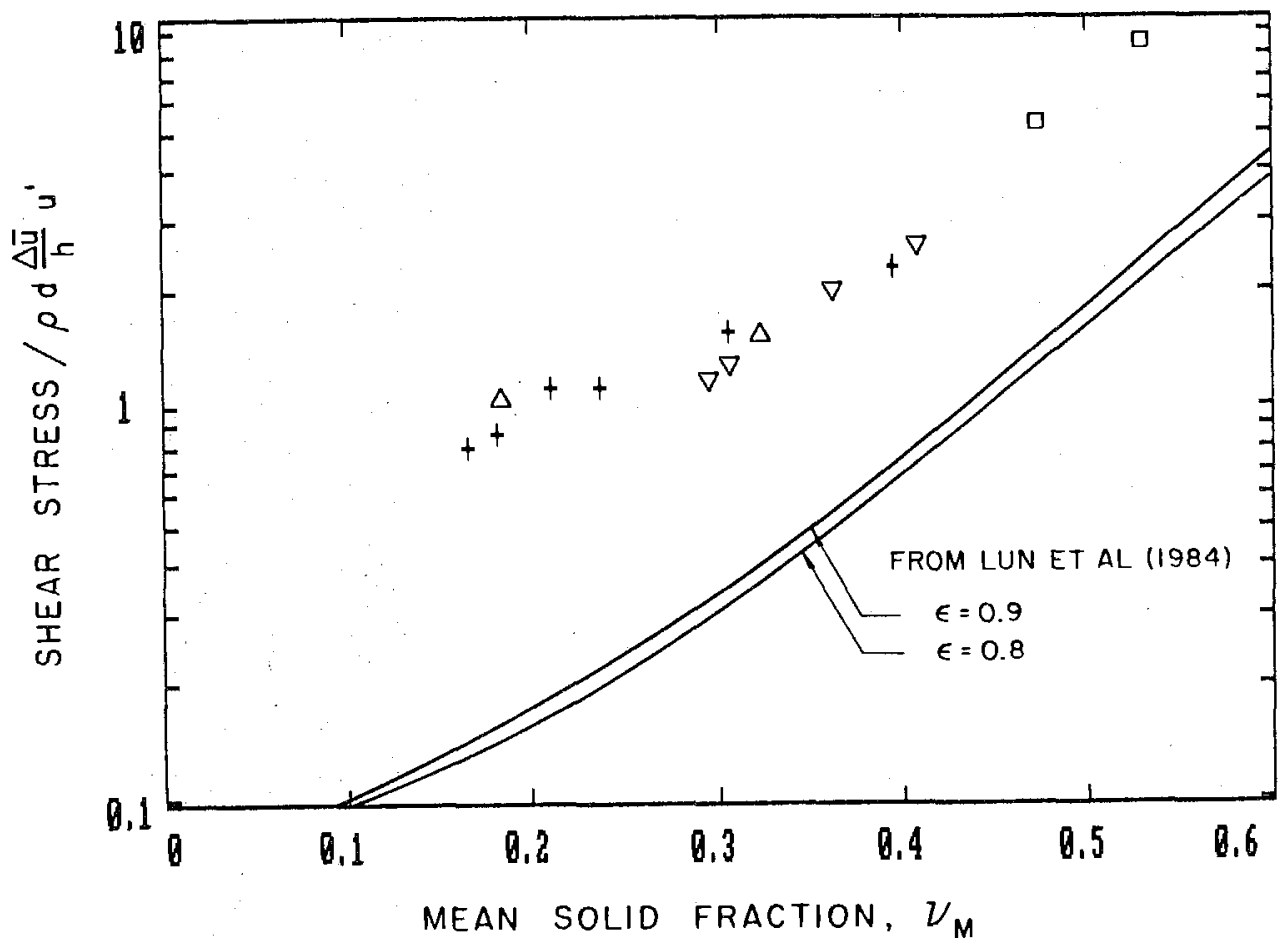

Figure 2. The shear stress function, $h \tau_{S} / \rho d \Delta \bar{u} u^{\prime}$, against mean solid fraction, $\nu_{M}$. Data points $h_{o}=1.59 \mathrm{~cm}$ and moderately dirty surface:,$+ h_{o}=1.59$ cm and clean surface: $\triangle, h_{o}=2.54 \mathrm{~cm}$ and moderately dirty surface: $\nabla, h_{o}=5.08 \mathrm{~cm}$ and moderately dirty surface: $\square$. Also shown are the analytical results of Lun et al [1984] for coefficients of restitution, $\epsilon$, of 0.8 and 0.9 .

and flow depths (gate height). It should be noted that if, instead of $\Delta u / h$, the mean bulk shear rate, $d \bar{u} / d y$, from the sidewall velocity profiles were employed in evaluating $g_{S}(\nu)$ then the ordinates in figure 2 would only be altered in a minor way and the correlation would still hold.

The theoretical prediction from Lun et al (1984) for a simple shear flow is also presented in figure 2; though Lun et al present results for different coefficients of restitution, $\epsilon$ between the particles the variation of the results with this parameter is small for the expected range of $\epsilon$ relevant to the experiments. The discrepancy between the theoretical and experimental results of figure 2 is substantial. Part of the reason for this may be that the channel flow is far from being a simple shear flow even though, according to the sidewall velocity profiles, the shear rate is relatively constant over the bulk of the depth.

The corresponding normal stress function $g_{N}(\nu)=\tau_{N} / \rho\left(u^{\prime}\right)^{2}$ is presented in figure 3 . Unlike the shear stress data it appears to be less well correlated; in fact, one can distinguish different lines for different gate heights, $h_{o}$, and surface frictional qualities. 


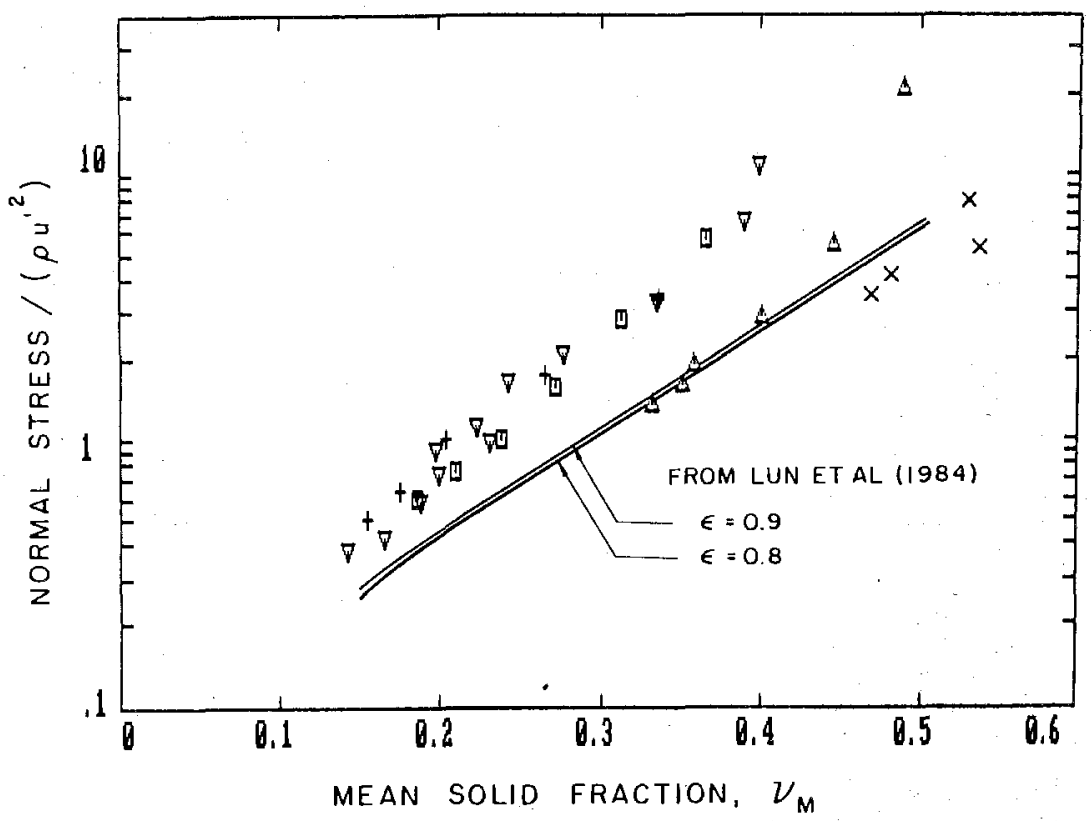

Figure 3. Normal stress function of $\tau_{N} / \rho\left(u^{\prime}\right)^{2}$ plotted against mean solid fraction, $\nu_{M}$ Data points $h_{o}=1.59 \mathrm{~cm}$ and clean surface: $\square, h_{o}=2.54 \mathrm{~cm}$ and clean surface: $\triangle, h_{o}=1.59 \mathrm{~cm}$ and moderately dirty surface: $\nabla, h_{o}=5.08$ $\mathrm{cm}$ and moderately dirty surface: $\times, h_{o}=1.59 \mathrm{~cm}$ and dirty surface: + . Also shown are the analytical results derived from Lun et al [1984] for coefficients of restitution, $\epsilon$ of 0.8 and 0.9 .

On the other hand it more closely agrees with the theoretical predictions of Lun et al, especially at the lower solid fractions where the theory is expected to be more accurate and the components of the fluctuations more isotropic.

The friction coefficient or ratio of $\tau_{S} / \tau_{N}$ is shown in figure 4 and is remarkably constant and Coulomb-like. This implies that a Coulomb frictional condition is appropriate at the wall and therefore that the relations (1) and (2) cannot both hold at the solid boundary.

It is therefore important to examine one of the most important constituents of the theories, namely the ratio of the fluctuating velocity to the shear rate, $d \bar{u} / d y$. From the current experiments we estimate this parameter as $S=d \Delta u / h u^{\prime}$ and values of this quantity are plotted against mean solids fraction in figure 5 .

Note that the data for various depths and surface qualities do not reduce to a single correlation, $S\left(\nu_{M}\right)$. The analysis of Lun et al [1984] predicts that, in a simple shear flow with a given coefficient of restitution, $S$ is a function only of $\nu$. The fact that the data do not reduce to a single function $S(\nu)$ implies that the channel flow is not a simple 


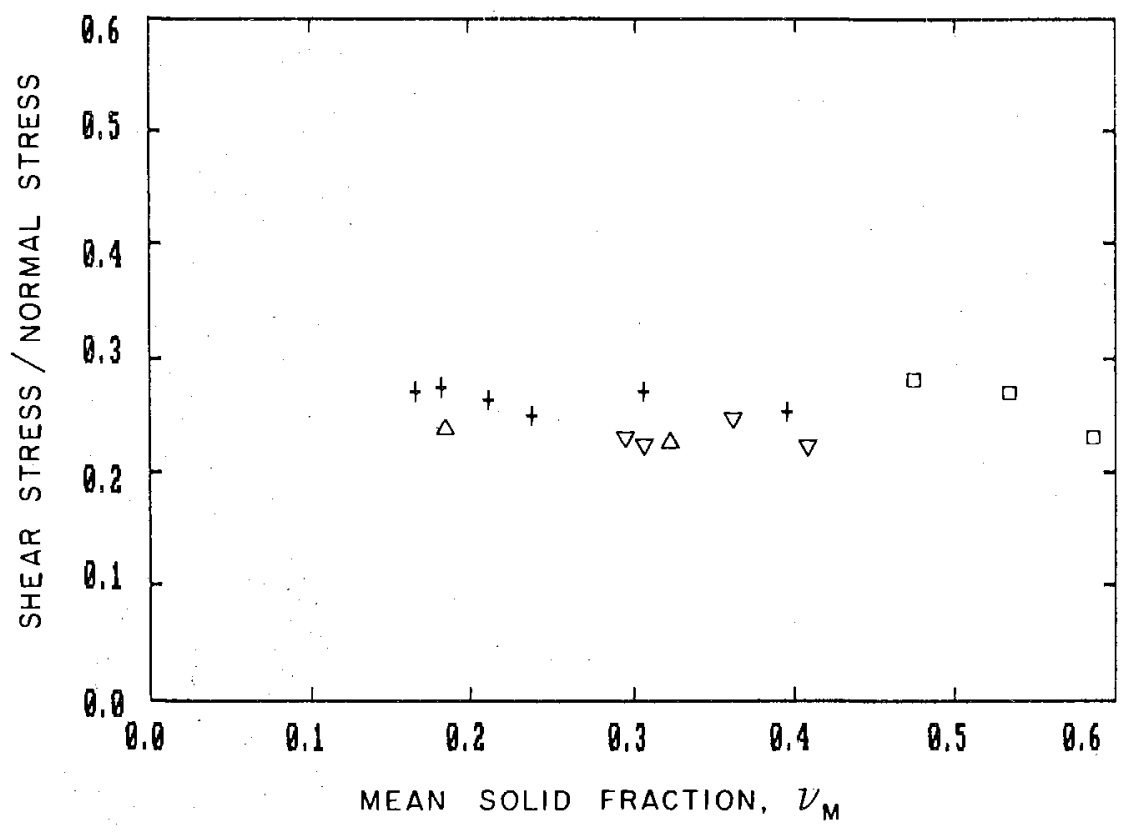

Figure 4. The friction coefficient of ratio. $\tau_{S} / \tau_{N}$ as a function of mean solid fraction, $\nu_{M}$. Data as in Figure 2.

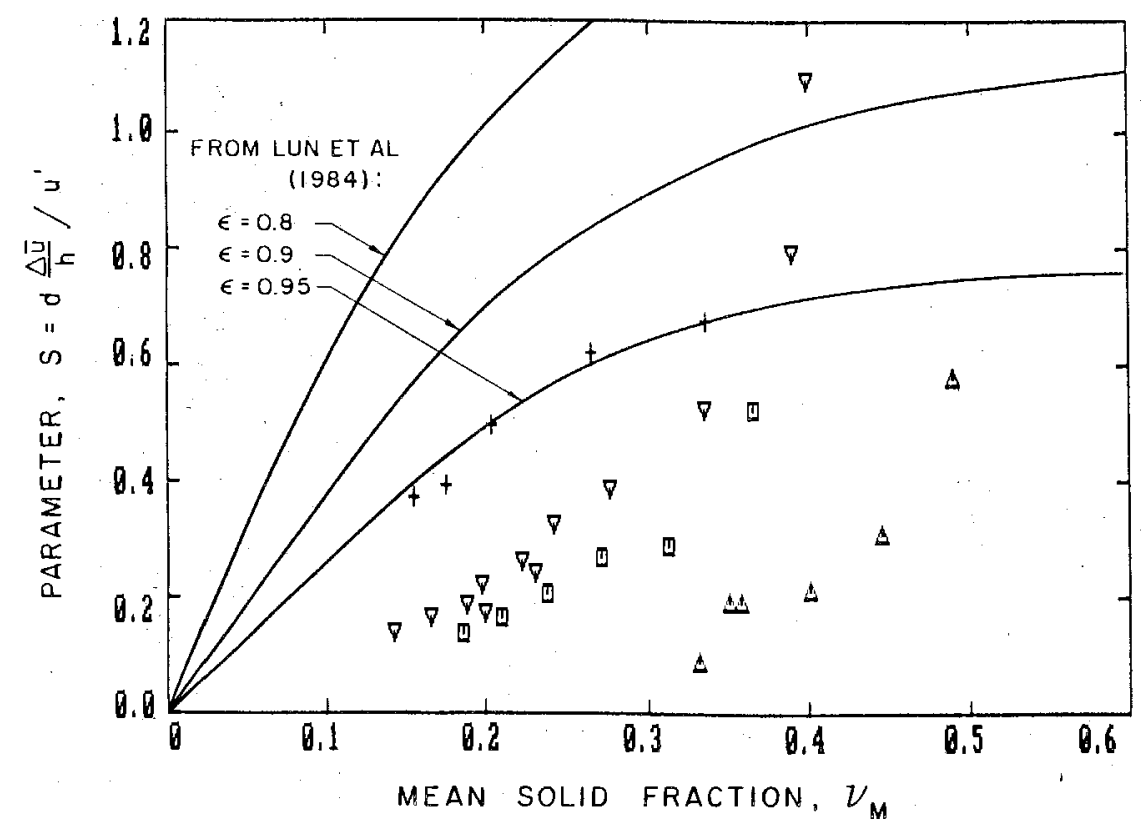

Figure 5. The parameter, $S=d \Delta \bar{u} / h u^{\prime}$, plotted against mean solid fraction, $\nu_{M}$. Point notation as in Figure 3. Also shown are analytical results derived from Lun et al [1984] for coefficients of restitution, $\epsilon$, of $0.8,0.9$ and 0.95 . 
shear flow. It would appear that the imposition of a Colomb boundary condition caused substantial departure from the simple shear flow relations in the neighborhood of the solid surface. This may involve significant anisotropy and/or conduction of granular heat in the boundary layer close to the wall.

\section{CONCLUDING REMARKS}

Experiments in inclined channels were designed to measure the granular temperature as well as the normal and shear stresses in a series of rapid flows of granular materials. The objective was to evaluate some features of existing rheological models for granular materials. The results of Lun et al (1984) were chosen as representatives of the latter.

Preliminary results indicate that the shear stresses on the base of the channel when normalized by dividing by the density, the mean shear rate and the fluctuating velocity do appear to reduce to a single function of the solid fraction. This function is, however, significantly larger than predicted by the theory. On the other hand, the normal stress when normalized by $\rho\left(u^{\prime}\right)^{2}$ seems to yield values which depend not only on the solid fraction but also on the base frictional quality and other factors. The same is true of the parameter $S$ or ratio of the shear velocity to the fluctuating velocity. The friction coefficient is quite constant and independent of the solid fraction. Thus the flow near the solid surface is not a simple shear flow but departs from this state to satisfy a Coulomb frictional condition. Experiments are continuing in an attempt to clarify these relationships.

\section{ACKNOWLEDGEMENT}

The authors are most grateful for the help given by S. Wakayama, D. Pillsbury, and C. Bertani during the conduct of this research. This work was supported by the National Science Foundation under Grant No. MEA 8400322. Some support from Union Carbide is also gratefully acknowledged.

\section{REFERENCES}

1 Augenstein, D.A., and Hogg, R., 1978, "An Experimental Study of the Flow of Dry Powders on Inclined Surface," Powder Technology, 19, p.205.

2 Bailard, J., 1978, "Experimental Study of Granular - Fluid Flow, Ph.D Thesis, University of California, San Diego.

3 Campbell, C.S. and Brennen, C.E., 1985a, Computer simulation of granular shear flows, J. Fluid Mech. Vol. 151, p. 167.

4 Campbell, C.S. and Brennen, C.E., 1985b, Chute flows in a two-dimensional granular shear flow, J. Fluid Mech, Vol. 52, p. 172.

5 Campbell, C.S. and Gong, A., 1986, The stress tensor in a two-dimensional granular shear flow. J. Fluid Mech. Vol. 164, p. 107.

6 Craig, K., Buckholø, R.H., and Domoto, G., 1986, "An Experimental Study of the Rapid Flow of Dry Cohension Less Metal Powder," J. Appl. Mech., Vol. 53, p. 935. 
7 Hanes, D.M., and Inman, D.L., 1985, "Observations of Rapidly Flowing Granular Fluid Materials," J. Mech., Vol. 150, p. 357".

8 Jenkins, J.T. and Savage, S.B., 1983, A theory for the rapid flow of identical smooth nearly elastic particles, J. Fluid Mech. Vol. 130, p. 1987.

9 Lun, C.K.K., Savage, S.B., Jeffrey, D.J. and Chepurniy, N. 1984, Kinetic theories for granular flow: inelastic particles in Couette flow and slightly inelastic particles in a general flowfield, J. Fluid Mech. Vol. 140, p. 223.

10 Patton, J.S., Sabersky, R.H., and Brennen, C.E. 1986 Convective heat transfer to rapidly flowing granular materials. Int. J. Heat Mass Transfer, Vol. 29, No. 8, p. 1263.

11 Sayed, M., and Savage, S.B., 1983, "Rapid Gravity Flow of Cohesionless Granular Materials down Inclined Chutes," J. Appl. Math. Phys., Vol 34, p. 84.

12 Savage, S.B. 1979 Gravity flow of cohesionless granular materials in chutes and channels, J. Fluid Mech., Vol.92, p. 53.

13 Savage, S.B. and McKeown, S., 1983, "Shear Stress Developed During Rapid Shear of Dense Concentrations of Large Spherical Particles between Concentric Cylinders," J. Fluid Mech., Vol. 127, p. 453.

14 Walton, O.R., 1984, Computer simulation of particulate flow. Energy and Tech. Rev, Lawrence Livermore Lab., May 1984, p. 24. 